\title{
Physically locating the present: a case of reading physics as a contribution to philosophy
}

\section{Introduction}

What is "integrated" history and philosophy of science? How do you do it? Why is it interesting? Why is it useful? Recent scholarship presented under the banner of "\&HPS" displays a rich variety of ways of doing "\&HPS": there are many different things that we are trying to achieve, and many different ways of going about it. In this paper I argue that reading history of physics as a contribution to history of philosophy is important for contemporary philosophy of physics, offering in the process an example of one kind of activity we might engage in under the broad umbrella of "\&HPS".

My argument centers around a particular case: special relativity versus presentism. By means of resources drawn from reading aspects of Newton's work as contributions to philosophy, I argue that there is in physics an alternative way to approach what we mean by the "present", distinct from ideas familiar from special relativity such as a preferred spacetime foliation. I offer this proposal for further philosophical investigation, and claim that if it is to be refuted empirically then this will require resources that go beyond those available in special relativity. Section 2 explains what I mean by the phrase "reading physics as a contribution to philosophy". Section 3 sets out the dispute between special relativity and presentism, as it is standardly understood. Section 4 outlines the resources that I will draw on from reading Newton, and then in Section 5 I deploy these resources to motivate an alternative version of presentism. My goal is to offer an example of reading history of physics through the eyes of a philosopher, as a contribution to philosophy, and to offer it as an example of one fruitful way in which we pursue integrated HPS. 


\section{Reading physics as a contribution to philosophy: the general project}

Famously, the divide between philosophy and physics is modern, originating in the philosophical developments of the seventeenth century. While Descartes's Principia Philosophiae of 1644 continues to be studied by philosophers, Newton's Philosophae Naturalis Principia Mathematica, published in 1687, does not appear on the standard reading list for early modern philosophy and is today thought of by most philosophers as a text in physics. Nevertheless, physics and philosophy from then to the present continue to have overlapping domains of investigation: space, time and matter being the most obvious examples. Just as philosophy begins from everyday experience and investigates our concepts of space, time and matter, clarifying and changing them along the way, so too does physics. Restricting our attention to time, the point is that there is no "every day" concept of time that we can make use of philosophically that is independent of the scientific concept: time as investigated by physics just is time as investigated by philosophy. That said, the questions that a physicist seeks to answer may not be those that interest the philosopher, and it may not be readily apparent how to bring the work of physicists to bear on the concerns of philosophers. As philosophers of physics well know, reading physics as a contribution to philosophy is not just a matter of reading physics.

There are different ways to engage with physics philosophically. Very often, philosophers of physics work on "interpretations" of theories in physics: they begin with a piece of theoretical physics and work their way from this towards philosophical questions. Here is an example of a philosopher of physics describing this work: ${ }^{1}$

Physics provides theories which typically consist of a mathematical formalism and some procedures for applying that formalism to particular concrete situations. But both the formalism and the procedures may admit of alternative ontological interpretations. It may not be clear, for

\footnotetext{
${ }^{1}$ Maudlin, in Loux and Zimmerman (eds.), 2003, pp. 461-2. I quote Maudlin here not as an example of a philosopher of physics who endorses this approach, but because of the clear description he gives of it.
} 
example, which part of the mathematics corresponds to real physical magnitudes and which is an artefact of arbitrary choices of units of gauges. It may not be clear which mathematical models represent real physical possibilities, and which do not. And it may not be clear which pairs of mathematical models represent the same physical situation. All of these problems confront even the philosopher who tries to take, for example, the Theory of Relativity 'at face value'.

This is one possible approach, and there is important conceptual work to be done here, but I do not think it is the most profound philosophically. An alternative is to begin with the deepest of our philosophical questions, and to use the development of physics read as a contribution to philosophy to explore how these questions are transformed, re-worked, addressed, and sometimes rendered non-questions. One does not "help oneself" to a philosophically shallow formalism, and then attempt to do philosophy: one sees physics as a part of the history of philosophy, and engages it on those terms. This is the kind of historical approach that I advocate. Not only must the philosopher read developments in physics as contributions to the projects in which the physicist is engaged, but also she must read between the lines, with the questions of a philosopher as her guide, re-reading developments in physics in that light.

As an example, consider again Newton's Principia. This is a very difficult book to read, and is most especially difficult to read as a philosophical text: Newton does not explain the whys and wherefores of what he is doing, let alone the philosophical context and ramifications. It is not surprising that philosophers of the period (Leibniz, Locke, Berkeley, Hume, Kant and so forth) did not see all of the philosophical moves and their implications, but this does not mean they are not there. We have now had over 300 years to begin to understand the Principia as a philosophical text. To re-visit it as a philosophical text of its time, and to weave what we learn into our philosophical history up to the present day, is to undertake the kind of work that I have in mind.

The re-reading and re-telling of history of philosophy plays a vital role in contemporary philosophy: we better understand our own philosophical questions and the philosophical space in which 
we work by paying attention to how we got here. So too I maintain that the re-reading and re-telling of history of physics, read as a contribution to philosophy, is important for contemporary philosophy of physics. In this paper I make a case for this claim by means of an example: special relativity and "presentism".

\section{Special relativity versus presentism}

Within philosophy of physics, there is a clear consensus that the advent of special relativity spells the death of "presentism"2 as a respectable philosophical position within philosophy of time. All that is needed to reach this conclusion is to "take special relativity seriously", which means understanding the critique of our concepts of space and time that it involves (I give more details below) and then accepting the theory as a complete account of spatiotemporal structure. This, in turn, is to accept a methodology and epistemology that emphasizes sensitivity to empirical considerations in theories and theorizing. Here is not the place to elaborate this methodology and epistemology, but I am persuaded by it and I take special relativity seriously. Nevertheless, through attention to history of physics, I argue that the debate over presentism should not yet be considered closed.

Special relativity (thus "taken seriously") is relevant to various aspects of our "experience of time”. To investigate this, we first take the Minkowski structure of spacetime and look to see which things have correlates in that structure and which things don't. Then we decide whether special relativity explains, explains away, or is just silent about that feature of our "experience of time". For example, temporal intervals between events, perhaps as measured by clocks, are explained: the structure of Minkowski spacetime provides an absolute spacetime interval between any pair of events, and this spacetime interval can be decomposed into a spatial interval and a temporal interval relative to a frame

\footnotetext{
${ }^{2}$ By "presentism" I mean positions in philosophy of time which accord a privileged ontological status to the "present", and I will say in more detail what I take the presentist thesis to be in a moment.
} 
of reference. So we can find within the structure of Minkowski spacetime elements that enable us to explain temporal intervals. The directionality of time, on the other hand, is something about which special relativity is silent. A direction of time is compatible with Minkowski spacetime and is usually built into it: for example, we stipulate that one half of the lightcone structure is the forward lightcone of an event, and the other half is the backward lightcone. Since this forward/backward distinction is put in by hand it's not clear that there is any explanatory work being done by the structure of Minkowski spacetime when it comes to a direction of time. We put it in because other areas of physics seem to indicate we need it, and we do no violence to the conceptual developments wrought by special relativity if we stipulate that one half of the lightcone structure is forward and the other backward. Special relativity is simply silent on this issue. When it comes to a flow of time there's no correlate in the structure of Minkowski spacetime. Compare this with the case of direction: in that case we can label the lightcones "forward" and "backward" if we choose, but in the case of flow there is nothing in the structure for us to label that could in any way correlate to a "flow". The standard philosophy of physics conclusion is that the flow of time is to be explained away as an illusion that has no counterpart in the spatiotemporal structure of the world.

My view is that our explorations of our spatiotemporal concepts through developments in physics can be used to help us distinguish between aspects of our "experience of time" which have an explanatory basis in the spatiotemporal structure of the world and which are to be "explained away".

One temporal thesis that should be explained away, according to standard philosophy of physics, is so-called "presentism". To see the clash between special relativity and presentism, consider the following two premises: ${ }^{3}$

(P1) All and only things that exist now are real.

\footnotetext{
${ }^{3}$ See Stein (1968, p.18) who notes that adoption of both (P1) and (P2) leads to "the interesting result that special relativity implies a peculiarly extreme (but pluralistic) form of solipsism."
} 
(P2) Special relativity is a complete account of spatiotemporal structure.

The first premise expresses a version of "presentism". It says that what is real is what exists right now: this is what there is. Things in the past aren't real (they don't exist any more); things in the future aren't real (they don't exist yet). What there is, is what is present. "Now" bears a great deal of ontological weight. The second premise says that there are no good reasons for adding anything to the account of space and time found in Einstein's special theory of relativity: it's complete.

The problem for people attracted to presentism is that $(\mathrm{P} 2)$ seems to be incompatible with (P1), for reasons that I'll come to immediately below. The dominant view amongst philosophers of physics is that we should reject (P1) on the grounds that "the present" as it is being used in (P1) is not empirically well-founded. Instead, we should adopt the so-called "block universe", a four-dimensional structure where everything that has ever existed, and will ever exist, is all equally real.

The reason for this conclusion is familiar from the literature on space and time. Given an event e1 in spacetime, e1 is "now" relative to itself, but there is nothing within the structure of special relativistic spacetime that determines which events spatiotemporally distant from e1 are also "now" relative to e1. There's no preferred way to "join the dots". You can conclude this directly from conventionality of simultaneity, in which case any "joining of the dots" in planes of simultaneity is an addition, going beyond the content of special relativity. Or you can get there via relativity of simultaneity: adopt the Einstein synchrony convention, note that different planes of simultaneity make different determinations of which events are "now" relative to e1 and which are not, note that picking one of these - a preferred plane of simultaneity - goes beyond the content of special relativity, and so conclude that no other events are determinately "now" with respect to e1. Either way, the conclusion is that there's no preferred way to join the dots. There is nothing in the structure of spacetime that correlates with "now", and to add any such structure would be to fail to learn the hard-won lessons of 
special relativity. There is no "now" that can bear the weight of $(\mathrm{P} 1)$. We should reject it, focus our attention on (P2), and endorse the block universe: what special relativity gives us is the entire set of events, arranged in a four-dimensional block. There are lots of ways to finesse things, but this is the basic argument.

I am in agreement with the proponents of the "block universe" in this dispute with presentists, as I have set it up. This debate over presentism and its relationship to special relativity is long-since settled: one cannot adopt presentism as expressed by (P1) and also adopt (P2). ${ }^{4}$

\section{Reading physics as a contribution to philosophy: a specific case}

As noted above, my interest is in studying physics as a contribution to philosophy, and in reading history of physics as a contribution to history of philosophy. My starting point and motivation are philosophical questions, and my interest is in how developments in physics bear on those questions: how they answer them, change them subtlely - or perhaps drastically -, and perhaps even render them non-questions. To my surprise, while reading Newton in this way I stumbled into a very surprising pay-off in contemporary philosophy of physics, one which re-opens the debate between special relativity and presentism. While the relevance of Newton's work to philosophical questions concerning space, time and body is perhaps obvious (if not sufficiently well understood even now), the wider and deep significance of his work for epistemology and metaphysics is less obvious. Although Newton seldom addressed such issues explicitly, the moves that he makes are often directly relevant to them and show a deep understanding of and engagement with epistemological and metaphysical questions. Recent Newton scholarship (see for example the papers in the Janiak and Schliesser, 2012) challenges the widespread view that Newton can be disregarded as a philosopher in his own right. For example, the sophistication of Newton's methodology and its bearing on epistemology is being brought into new

\footnotetext{
${ }^{4}$ This is not quite true: one could adopt Stein's (1968, p. 18) “pluralistic extreme solipsism” (see section 5, below).
} 
focus by the work of Smith $(2001,2002)$ and Harper (most recently his 2012). In my work on Descartes and Newton, I have argued that Newton makes important contributions to metaphysical questions concerning unity and change (Brading 2011, 2012, forthcoming). While seemingly unrelated to special relativity and presentism, the approaches to unity that can be found in Newton's work turn out to have unexpected relevance for our understanding of special relativity and presentism. Or so I shall argue in the remainder of this paper.

Perhaps the most familiar principle of unity found in Newton's work is space and time. Absolute space and time are the framework within which the dynamics of the Principia unfold, and within which all the material beings and spirits of this world are contained. In the manuscript 'De Gravitatione' (Newton, 2004), the status of space and time is robustly metaphysical. Space and time are emanations of God, and every being is somewhere and somewhen. For our purposes, the important point is that, for two things to belong to the same world, it is necessary and sufficient that they be spatiotemporally related: space and time are playing the role of a principle of ontological unity. We are used to thinking this way. Typically in contemporary philosophy of physics, spacetime provides the ontological unity of the world: what makes this material universe one universe is the shared spatiotemporal framework within which the matter is located.

A second principle of ontological unity found in Newton's work emerges from considerations of a different kind. A question that pervades all of my work is this: what is physics about and how does it come to be about whatever it is about? In the context of Descartes and Newton, one question this begets is "What are the bodies that are the subject matter of Descartes's (or Newton's, respectively) laws?" In considering Descartes's laws, Newton faced this question too, and he gave a very interesting answer. In the Principles Part II, Descartes offered an account of body that is famously incomplete. I have argued elsewhere (Brading 2012) that Newton made explicit appeal to the laws of motion in attempting to complete the account of bodies: he offered a law-constitutive account of bodies. On this account, the 
very constitution of the body as a body, in the most generic sense, depends on the laws: what makes it a body at all is, at least in part, that it satisfies Newton's laws. To see what this means, begin with the case of simple bodies free from interactions. The first law requires that such bodies conserve their quantity and direction of motion. From the law-constitutive perspective, the conservation of quantity and direction of motion is (at least partially) constitutive of the entities that are the subject-matter of the first law. Each such body is a body, and is one, in virtue of conserving its quantity and direction of motion. Now think about the extension to composite systems. The role of Newton's second law is to determine how our lone body of the first law will change its state under the influence of external forces. Such forces arise through the interaction of bodies among themselves. The role of the third law is to determine the behaviour of interacting bodies, behaviour that must be consistent with the first law continuing to hold for the collection of interacting bodies taken as a whole. From the law-constitutive perspective, such a collection of interacting bodies forms a composite whole in virtue of conserving its total quantity of motion and its overall direction of motion (the direction of motion of the center of mass of the collection). Thus, conservation of quantity and direction of motion can be understood as a principle of unity for a composite system, when that system as a whole is free from external interactions.

One further conclusion (from Brading 2011) is important for our present purposes. As noted above, by means of his third law Newton provides a rule which determines the behavior of interacting bodies, such that the motion of the component bodies is determined uniquely and quantifiably. This is the means by which we extend the law-constitutive approach to the component bodies of a composite system: Newton's laws give necessary conditions for something to be a part of a composite system, and sufficient conditions for those parts to be determinate. As such, the law-constitutive approach delivers a principle of unity for the parts of composite systems.

This review has been brief, but the upshot can be summarized as follows. We are being offered a principle of ontological unity that applies across the board to simple things, composite systems, and 
component parts of those systems, and which applies all the way up to the material universe as a whole.

Moreover, it can be used to address the three key metaphysical problems associated with unity:

multiplicity, aggregation and composition. By multiplicity I mean such questions as "Does the world contain a multiplicity of genuine unities? And if so, what's the principle of unity?" By aggregation I mean "If the world contains a multiplicity of genuine unities, how do they together form a whole, rather than being merely a collection?" The members must stand in relations to one another, and those relations must be necessary and sufficient for the collection to form a unity. And by composition I mean "Are there any composite wholes less than the world as a whole?" While I am not for a moment claiming that these questions of unity were Newton's target, I do think he was explicitly concerned with what the bodies are that are the subject matter of physics, and I do want to say that the moves he makes in addressing that question are directly relevant to these questions concerning unity. Moreover, these were certainly live questions of the time, and Newton was explicitly concerned with issues of divisibility and atomism.

This is an example of where Newton's work in physics is directly relevant to philosophical questions that long pre-date Newton and continue to occupy some philosophers today. Yet you will not find any reference to Newton's work in the history of philosophy that we tell ourselves today on this topic. My suggestion is that there is much to be gained philosophically by re-visiting Newton's work read as a contribution to philosophy.

I have not argued for the law-constitutive approach to unity here, nor for its basis in Newton's work. For that I refer to Brading $(2011,2012$, forthcoming). Rather, my goal in this paper is to argue that reading history of physics as a contribution to history of philosophy in this way is important for contemporary philosophy of physics, and to do so by using the insights gained from this reading of Newton in the context of the debate over special relativity versus presentism. 


\section{Special relativity and presentism again}

Presentism as expressed in (P1) attempts to ground the unity of what exists, of what is real, in simultaneity: all and only things that exist now are real. If the "now" of a given real thing extends to other things, then those other things are also real: the unity of the real rests on simultaneity. Absolute simultaneity, being spatially extended across the entire spatial extent of the universe, ensures that "what is real" (according to (P1)) is also spatially extended across the entire spatial extent of the universe. Problems for presentism arise when "now" is not spatially extended, because then what is real, given (P1), is not spatially extended either. In the case of special relativity, applying (P1) means that what is real relative to event $\mathrm{e} 1$ is what is now relative to event $\mathrm{e} 1$, and that means that only $\mathrm{e} 1$ is real relative to e1. ${ }^{5}$ A background assumption here is the use of space and time as an ontological principle of unity: "now" is being used as the ground of what is real.

Advocates of the block universe also use space and time as an ontological principle of unity, but do so in a different way. The four-dimensional structure of Minkowski spacetime serves as the container in which all material events take place. Thus, both the advocate of special relativity and the defender of presentism share a common assumption in using space and time as an ontological principle of unity. Both adopt Newtonian option 1 .

The lesson from Newton (from section 4, above) is that there is at least one other option available: the law-constitutive approach to ontological unity. What would happen to the relationship between special relativity and presentism if we dropped the assumption that space and time should play the role of an ontological principle of unity? What would happen if we adopted a law-constitutive approach instead?

First, let's think about the status of space and time. If we don't need them as a principle of ontological unity, what role do they play? We can answer this question by addressing why we set out a

\footnotetext{
${ }^{5}$ This is Stein's "pluralistic extreme solipsism" (Stein, 1968).
} 
big arena of space and time when we're doing physics. In mechanics, for example, we want to know what the outcome of a collision will be, before it happens, based on knowledge of events prior to the collision. Quite generally, one thing we're doing is extending our knowledge of events to times and places distant from the here and now. Space and time play a theoretical role, as we try to extend our epistemic reach beyond the here-now, stitching our predictions, retrodictions, and knowledge of past events together into a single whole. Thinking of things in this way, space and time provide an epistemic principle of unity: they provide the framework in which we organize our knowledge of the not-here and/or not-now. There is no necessary inference from this epistemic role for space and time to ontological conclusions. Approached this way, the spatiotemporal structure of Minkowski spacetime should be understood epistemically, and "taking special relativity seriously" as required by (P2) means adopting this structure as the framework in which we organize events for epistemic purposes. We can be committed to special relativity as a complete account of the best spatiotemporal structure we have for such purposes, while remaining cautious about drawing ontological conclusions on this basis. Taking special relativity seriously as an epistemic principle of unity means that special relativity is the best way to organize our knowledge that reaches beyond the here-now.

Second, let's think about (P1), the presentist thesis. (P1) explicity appeals to "now" as the ground of what is real. Using the suggestion drawn from our considerations of Newton's work above, we reject this role for time (or for space and time more generally). Instead, we begin from the premise that what exists (what is real) are the genuine unities, and we offer a law-constitutive approach to determining what these entities are.

In section 3, above, I discussed the way in which presentism, and indeed our "experience of time" more generally, can be explored within the context of Minkowkski spacetime: we take the Minkowski structure of spacetime and look to see which things have correlates in that structure and which things don't. Presentism proved problematic because there is nothing within the structure of 
Minkowski spacetime that could be correlated with "now". Having adopted the law-constitutive approach, we can proceed in the same way: is there anything in the structure of physics thus construed that could be interpreted as the "present"?

Here is a proposal for how to develop a version of presentism within the law-constitutive approach: identify the "present" of any given unity with the spatiotemporal region that is dynamically necessary to sustain it. Thus, what is "present" depends on what is real (the genuine unities), and what is real depends on the dynamical laws. In the traditional approach to presentism, illustrated by (P1), space and time are taken as prior, and we seek to identify a feature of that spatiotemporal structure (the present) to ground what is real. In the approach being proposed here, the order of priority is reversed: we first determine what is real (via the law-constitutive approach) and seek features of this as the ground of what is present.

The proposal is that the present is a spatiotemporal region of whatever size is needed to sustain the dynamical system in question. It might turn out that this is the entire spatiotemporal history of the universe. If this is the case, we are back to the block universe and there is nothing in the structure of the physical theory in question that can be correlated with anything we would want to call "present". But if it turns out that the size of the spatiotemporal region necessary to sustain a particular dynamical system is less than the entire spatiotemporal history of the universe, then this calls the ontology of a block universe into question. Moreover, if it is less than the entire spatiotemporal history of that particular system, then we have a "present" for that system that is distinct from its history. This is a version of presentism that follows naturally from the law-constitutive approach to the entities that are the subjectmatter of physics. It does not "add" anything empirically unwarranted to the physics (as those who would add a preferred foliation to special relativity want us to do) and takes seriously the lessons of special relativity. For example, such a "present" is local, in being a spacetime region less than the extent of the entire "block". This comes about because being present is relative to the dynamical system in 
question: it is localized to the spatiotemporal region necessary to sustain that system and is not transitive among systems and their subsystems. ${ }^{6}$ Thus, the idea of a spatially global present common to all systems is not preserved in this version of presentism, but the rejection of a spatially global present is one of the central lessons of special relativity, one that any presentist should accept, so this is all to the good. Secondly, the "present" is a spatiotemporal region, and there is no attempt to provide a unique foliation of this region into "spatial" and "temporal" components: the present is extended in time just as it is extended in space, precisely because it is extended in spacetime. Again, this is exactly what we would expect given special relativity.

How might we re-formulate (P1) in the light of these considerations, and what would this form of presentism look like? How much of presentism, as understood by advocates of (P1), is preserved? We might try the following as a replacement for (P1): "For each and every thing, that thing exists only presently”. But that doesn't really say anything, so here is another attempt:

$(\mathrm{P} 1 *)$ For each and every thing, that thing exists only presently, where the spatiotemporal extent of that "present" is dependent on dynamics, and it is something to be determined empirically.

As we noted, this spatiotemporal extent might turn out to be the entire spatiotemporal extent of the universe, and having the entirety of the block universe labelled as the "present" doesn't seem to save anything that the presentist was hoping to save. So one more attempt seems in order, albeit a bit of a mouthful:

\footnotetext{
${ }^{6}$ Recall that on the law-constitutive approach being described here, we are not embedded in the same reality by means of a shared spatiotemporal structure, but by means of being parts of a composite dynamical system. Thus "my present" and "your present" need not be ontologically related to one another, nor need the "present" of one of my parts by ontologically related to the "present" of me as a whole, in order for us (and our law-constitutive parts) to belong to the same reality. Space and time do not play the role of an ontological principle of unity on this approach, that job is done by the laws.
} 
$\left(\mathrm{P} 1{ }^{* *}\right)$ For each and every thing that exists, that thing exists only presently, where for each thing the spatiotemporal extent of the present for that thing is dependent on the dynamics and is (a) less than the entire spatiotemporal evolution of the universe as represented in Minkowski spacetime, and (b) less than the entire spatiotemporal evolution of that thing as represented in Minkowski spacetime.

In order to avoid the block universe, all we need is (a). The second clause, (b), could be omitted, but should be included if we also deem it important to presentism that the "present" of any given thing is less than the entire spatiotemporal history of that thing.

It remains to be explored the extent to which the core of the presentists' intuitions can be captured or reinterpreted within this version of presentism. Insofar as (a) and (b) preserve something of the presentists' intuitions, they preserve those features that are compatible with special relativity and with "taking special relativity seriously" as (P2) in its epistemic version requires. I will be very interested to see whether those with presentist intuitions find the approach amenable.

This version of presentism seems to me to be philosophically viable, and it is respectable given special relativity. As such, it deserves to be explored as a philosophical option. The question of whether it is empirically viable must also be addressed: whether conditions (a) and (b) are satisfied in this, the actual world that we find ourselves in, is an empirical matter dependent on the details of the dynamics. My point is this: until we have answered the empirical question by exploring the details of the dynamics, the debate over the relationship between special relativity and presentism remains an open empirical question.

The case I hope to have made is that a discussion reasonably considered closed in contemporary philosophy of physics (whether presentism is a viable position) should not, in fact, be considered closed. The idea is that matter is spatiotemporal but it is not in space and time in the sense that space and time 
provide an ontological principle of unity for what there is. Whatever spatiotemporal properties dynamical systems have, including their spatiotemporal extents, are to be determined law-constitutively. I fully concede that this approach stands in need of significant further consideration and elaboration, but the idea I hope is clear. If the block universe is to triumph over presentism then further work must be done, work that draws on resources beyond those available in special relativity.

The availability of this position emerged from apparently unrelated work on reading Newton's work as contributing to philosophy. As such, this example is intended to support the broader thesis that reading history of physics as a contribution to history of philosophy is important for contemporary philosophy of physics.

To reinforce this latter point, I will end by making explicit a connection between this work and Harvey Brown's work on relativity. ${ }^{7}$ While much of Brown's book concerns special relativity, one of the central lessons for relativity theory is drawn in the final chapters on general relativity, where Brown pursues the question of the significance of the metric field $\left(g_{\mu v}\right)$. We can ask whether the role of $g_{\mu v}$ as the spacetime metric in the Einstein field equations can be ascertained from the theory or whether this interpretation must be put in independently by hand. Brown asks (2005, p. 160), "How does it come about that $g_{\mu \nu}$ is surveyed by physical rods and clocks, and that its null and time-like geodesics are associated with the world-lines of photons and massive particles respectively?" His proposal is that we should begin from the dynamics of the matter fields. His claim is that the Lorentz symmetries characterizing the structure of Minkowski spacetime originate in the dynamics of the matter fields, and that it is because of these dynamical laws, and because of the particular structure of the dynamical laws coupling the matter fields to $g_{\mu \nu}$ in general relativity, that $g_{\mu \nu}$ turns out to play the role of a spacetime metric. Thus, on Brown's approach, the matter fields have spatiotemporal characteristics, but they do not inherit them from a $g_{\mu \nu}$ already independently interpreted as the spacetime metric tensor. Rather, the

\footnotetext{
${ }^{7}$ Brown (2005). While I did not make this connection explicitly in my talk at the \&HPS4 conference, the ever-astute John Norton was quick to spot it.
} 
interpretation of $g_{\mu v}$ as a spacetime metric depends upon a detailed understanding of the dynamics of the matter fields.

This approach to the relationship between dynamics and spacetime structure would be a natural candidate as a starting point for someone wishing to pursue a law-constitutive approach to presentism, because it reverses the usual priority of spacetime over matter allowing for the possibility that whatever spatiotemporal framework we make use of in theorizing is ontologically dependent upon the dynamical laws for matter.

Even were this approach to succeed, the crucial question would remain of whether there are genuine subsystems of the world, as picked out by the dynamical laws, that are smaller in spatiotemporal extent than the entirety of spacetime. Only if there are such can the version of presentism offered here survive. But that is an empirical question, dependent on the details of the dynamical laws, and presentism in this form remains an empirical hypothesis yet to be explicitly refuted.

\section{Conclusions}

I have offered an example of one way in which integrated HPS is practiced: history of physics is read as a contribution to history of philosophy, framed and motivated by questions of philosophy. I have sought to show that, for one particular example (presentism and special relativity), the result of engaging in this activity is fruitful for contemporary philosophy of physics. This fruitfulness consists in pointing out a philosophical option open to would-be presentists who "take special relativity seriously", an option which can be connected to a current debate in philosophy of physics over the status of the spacetime metric and its relationship to matter fields. As a philosopher of physics, to me the "block universe" is the familiar mainstream position, to be arrived at directly from considerations of special relativity alone. I was most surprised to come upon a version of presentism that is compatible with "taking special 
relativity seriously". Of course, it will not preserve all the desiderata of contemporary metaphysical presentists, but we can explore what philosophical work this version of presentism can be made to do. The version of presentism that I offer here may well get refuted empirically, but any such refutation will necessarily draw on resources in physics beyond those that special relativity alone can offer. That is a lesson that I would not have come upon were it not for my work in integrated HPS.

\section{References}

Anstey, P., and Jalobeanu, D., ed. (2011) Vanishing Matter and the Laws of Nature: Descartes and Beyond, Studies in Seventeenth-Century Philosophy, Routledge.

Brading, K. (2011) 'On composite systems: Descartes, Newton, and the law-constitutive approach', in Anstey and Jalobeanu (2011) pp. 130-152.

-- (2012) 'Newton's law-constitutive approach to bodies: a response to Descartes', in Janiak and Schliesser (2012), pp. 13-32.

-- forthcoming, 'Three principles of unity in Newton', Studies in History and Philosophy of Science.

Brown, H. R. (2005) Physical Relativity (Oxford: OUP).

Harper, W. (2012) Isaac Newton's Scientific Method (Oxford: OUP).

Janiak, A., and Schliesser, E., ed.(2012) Interpreting Newton: critical essays (Cambridge University Press).

Loux, M. J., and Zimmerman, D. W. (eds.) (2003) The Oxford Handbook of Metaphysics (Oxford University Press).

Newton, I. (2004) 'De Gravitatione', in Philosophical Writings, ed. A. Janiak, Cambridge University Press, Cambridge, pp. 12-39.

Smith, G. E. (2001) “Comments on Ernan McMullin’s 'The Impact of Newton's Principia on the philosophy of science'” Philosophy of science, pp. 327-38. 
— (2002) "Newton's Methodology" in eds I. B. Cohen and G. E. Smith (ed.) The Cambridge

Companion to Newton, Cambridge University Press, Cambridge, 2002, pp. 138-173.

Stein, Howard (1968) “On Einstein-Minkowski Space-Time” Journal of Philosophy 65(1):5-23. 